ARTICLE

DOI: $10.1038 / s 41467-018-06956-x$

\title{
Phase-controlled Fourier-transform spectroscopy
}

\author{
Kazuki Hashimoto ${ }^{1,2}$ \& Takuro Ideguchi [i] ${ }^{1,3}$
}

Fourier-transform spectroscopy (FTS) has been widely used as a standard analytical technique over the past half-century. FTS is an autocorrelation-based technique that is compatible with both temporally coherent and incoherent light sources, and functions as an active or passive spectrometer. However, it has been mostly used for static measurements due to the low scan rate imposed by technological restrictions. This has impeded its application to continuous rapid measurements, which would be of significant interest for a variety of fields, especially when monitoring of non-repeating or transient complex dynamics is desirable. Here, we demonstrate highly efficient FTS operating at a high spectral acquisition rate with a simple delay line based on a dynamic phase-control technique. The independent adjustability of phase and group delays allows us to achieve the Nyquist-limited spectral acquisition rate over 10,000 spectra per second, while maintaining a large spectral bandwidth and high resolution. We also demonstrate passive spectroscopy with an incoherent light source.

\footnotetext{
${ }^{1}$ Department of Physics, The University of Tokyo, Tokyo 113-0033, Japan. ${ }^{2}$ Aeronautical Technology Directorate, Japan Aerospace Exploration Agency, Tokyo 181-0015, Japan. ${ }^{3}$ PRESTO, Japan Science and Technology Agency, Tokyo 113-0033, Japan. Correspondence and requests for materials should be addressed to T.I. (email: ideguchi@phys.s.u-tokyo.ac.jp)
} 
$\mathrm{F}$ ourier-transform spectroscopy (FTS) has provided solutions to countless chemical analysis problems in the fields of chemistry, pharmacy, medicine, biology, and physics including material science ${ }^{1-3}$. It enables the simultaneous identification of multiple molecular species via high-resolution broadband molecular spectra measured by a simple optical system consisting of a scanning Michelson interferometer and a broadband light source. The fact that this analytical method has long been widely used owes to the simple and robust autocorrelation-based working principle, enabling a variety of uses. For example, since it allows us to measure temporally incoherent light, FTS can be used as a passive spectroscopy technique with an external light source such as sunlight ${ }^{4}, 5$. However, FTS has been mostly used for measuring static samples because of the low temporal resolution due to the low scan rate of mechanical delay lines. This is incompatible for investigating fast phenomena at a higher temporal resolution, such as combustion processes which often require a measurement rate over $10 \mathrm{kHz}^{6,7}$. Rapid-scan FTS instruments typically operate at an improved temporal resolution only at a low spectral resolution, for example a scan rate of $1 \mathrm{kHz}$ with a resolution of $120 \mathrm{GHz}\left(4 \mathrm{~cm}^{-1}\right)^{8}$ or 77 $\mathrm{kHz}$ with a resolution of $360 \mathrm{GHz}\left(12 \mathrm{~cm}^{-1}\right)^{9}$. For a higher resolution applicable to gas-phase analysis such as $15 \mathrm{GHz}(0.5$ $\mathrm{cm}^{-1}$ ), the available scan rate significantly drops down to less than $10 \mathrm{~Hz}^{10,11}$ due to the lack of a rapid-scan long-range delay line. Step-scan FTS ${ }^{12}$ is also known to improve the temporal resolution but it works only for measuring repeatable phenomena, which significantly limits applications. Another type of broadband absorption spectroscopy technique, called timeresolved frequency comb spectroscopy (TRFCS) ${ }^{13,14}$, has been used for analyzing transient chemical phenomena of gaseous molecules with high spectral resolution. The scan rate of this technique is $250 \mathrm{~Hz}$, which is limited by the frame rate of the image sensor. The temporal resolution can be as high as $25 \mu \mathrm{s}$ through repetitive measurements, but it also works only for measuring repeatable phenomena.

A recent development in dual-comb spectroscopy (DCS) $)^{15,16}$, a mechanical-scan-free FTS technique based on mutually coherent laser frequency combs with slightly detuned pulse repetition rates, has elegantly solved the problem and shown a tremendous improvement on the measurement scan rate ${ }^{17-34}$. The superiority of this technique is its highest scan rate, i.e., Nyquist-limited scan rate, enabled by fully utilizing the Nyquist range, determined by half a repetition rate of the laser. In other words, DCS has been a unique FTS that enables the highest measurement rate, spectral bandwidth and resolution to be achieved under the Nyquistlimited trade-off constraint. This capability is owed to the adjustable difference in both the repetition rate and carrierenvelope offset (CEO) frequency between the combs. The former determines the down-conversion factor from the optical to radio frequency (RF) to achieve the highest possible scan rate, while the latter adjusts the position of the RF spectrum within the Nyquist range for avoiding aliasing. The latter capability has not often been clearly appreciated in the previous reports on DCS, but we emphasize that it assures DCS to be an efficient FTS technique. In return for this superior capability, DCS requires a more elaborate and sophisticated system than the conventional Michelson-type FTS because DCS is based on the cross-correlation measurement between the two lasers, for which mutual coherence is usually fragile. Furthermore, it is an active-type spectrometer, which requires light sources in the instrument, and is not suitable to be used in a passive manner ${ }^{20,22}$.

In this work, we revisit conventional Michelson-type FTS and develop a Nyquist-limited highly efficient FTS, called phasecontrolled FTS (PC-FTS), that allows us to arbitrarily adjust the scan rate, spectral bandwidth, and spectral resolution under a tradeoff relation among them. This approach uses a rapid-scan phase-control mechanism inspired by pulse shaping techniques ${ }^{35}$, that enables us to acquire interferograms continuously at a rate beyond $10 \mathrm{kHz}$. This is the highest scan rate achievable as a Michelson-type FTS, while avoiding spurious spectral distortions due to the aliasing effect. This is made possible by the arbitrary and independent adjustment of the group and phase delay added by the delay line, which can be understood as an analogy of the independent adjustment of the difference in repetition rate and CEO frequency in a dual-comb spectrometer. As a proof of concept demonstration, we measure broadband gas absorption spectra of hydrogen cyanide $\left(\mathrm{H}^{12} \mathrm{C}^{14} \mathrm{~N}\right)$ and acetylene $\left({ }^{12} \mathrm{C}_{2} \mathrm{H}_{2}\right)$ in the near-infrared region spanning over $1.8 \mathrm{THz}$ with a resolution of $11.5 \mathrm{GHz}$ at an acquisition rate of over $12 \mathrm{kHz}$ both with temporally coherent and incoherent light sources. Our system holds great promise for applications aimed at measuring nonrepeating phenomena at high-temporal-resolution such as a transient combustion process, or a large number of events at high-throughput such as large-area environmental monitoring.

\section{Results}

Principle of PC-FTS. A schematic of PC-FTS is shown in Fig. 1a. It consists of a broadband light source, a scanning Michelson interferometer, a single photodetector and a digitizer. Since Michelson-type FTS is an autocorrelation-based measurement, there is no requirement on the temporal coherence for the measured light source. In our experiment, a coherent modelocked laser or an incoherent amplified spontaneous emission from a super-luminescent diode (SLD) is used as a broadband light source. The scanning Michelson interferometer varies a time delay between the beams traveling along the reference and scan arms, so that an autocorrelation trace, called the interferogram, is obtained as a function of time by the photodetector. The digitized interferogram is Fourier-transformed to obtain a spectrum. By placing a sample in the optical path between the interferometer and the detector, its absorption feature can be encoded onto the spectrum. Our phase-controlled delay line consists of a dispersive element such as a diffraction grating, a focusing element and a scanning mirror, which are aligned in a $4 \mathrm{f}$ geometry such that each spectral component of the beam is focused at a different spot on the Fourier plane, where the scanning mirror is placed ${ }^{36,37}$. The reflected beam from the scanning mirror then passes through the optical elements in the $4 \mathrm{f}$ system and is retro-reflected by an end mirror. The $4 \mathrm{f}$ geometry ensures the beam travels back along the incident path for any facet-angles of the scanning mirror. Using a rapidly scanning mirror enables the system to function as a rapid-scan FTS. A detailed schematic of the PC-FTS is shown in Fig. 2 and the components used in the system are described in Methods section.

The working principle of PC-FTS is depicted in Fig. 1b. A slight inclination of the scanning mirror placed at the Fourier plane adds a linear spectral phase ramp to the broadband light that travels through the delay line. Here, we describe the system with a galvanometric scanner. Assuming the scanning mirror rotates clockwise at a constant angular frequency $\omega$, the added spectral phase $\phi(v)$ and group delay $\tau_{\mathrm{g}}$ may be described as:

$$
\begin{aligned}
& \phi(\nu) \approx-2 \pi c_{\mathrm{g}}\left(\nu-\nu_{0}\right) t \\
& \tau_{\mathrm{g}}=-\frac{1}{2 \pi} \frac{\partial \phi(\nu)}{\partial \nu} \approx c_{\mathrm{g}} t
\end{aligned}
$$

where $t$ denotes time, $v$ optical frequency, $v_{0}$ the optical frequency corresponding to the pivot position of the scanning mirror in the Fourier plane, and $c_{\mathrm{g}} \propto \omega / v_{0}$ the down-conversion factor, which is approximately a constant value in time. A full description of $c_{\mathrm{g}}$ is 
a

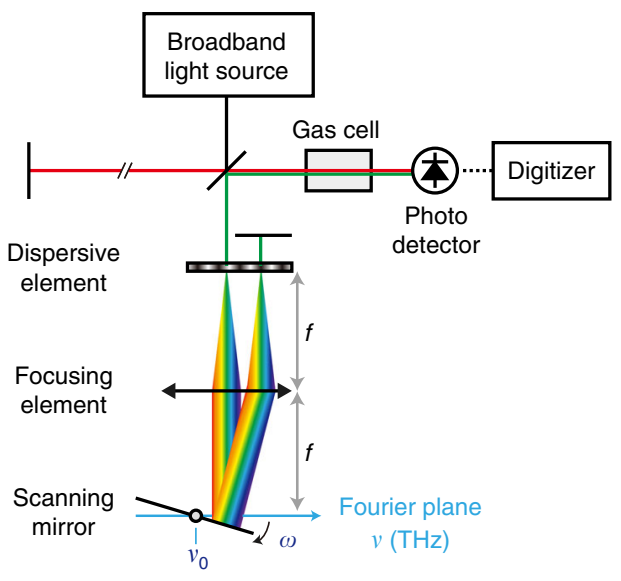

b

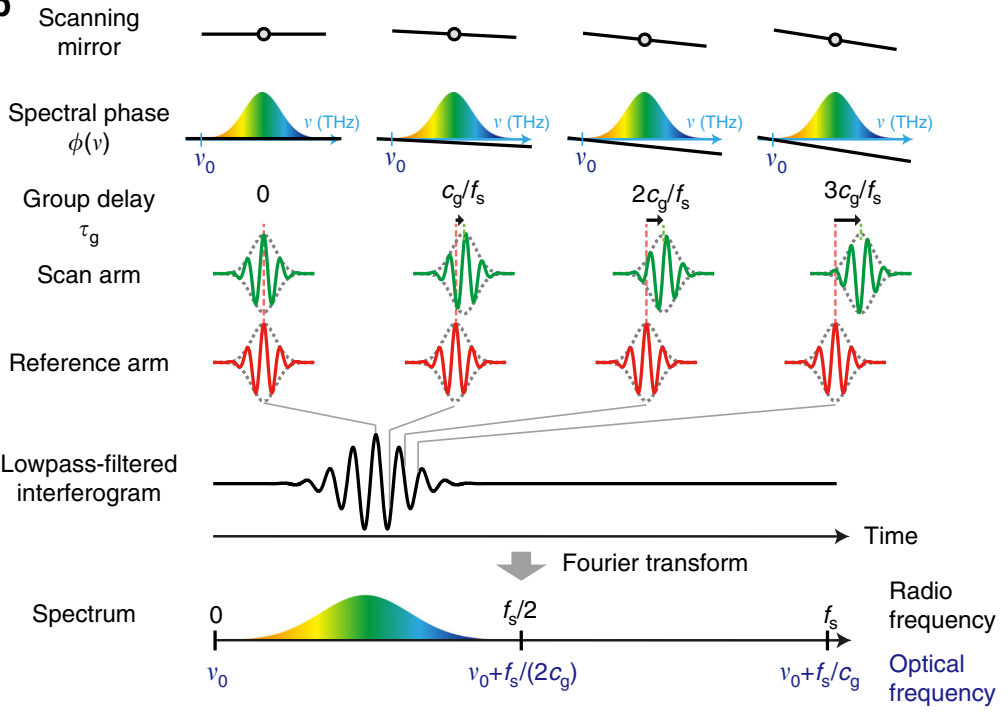

Fig. 1 Schematic and conceptual illustration of phase-controlled Fourier-transform spectrometer. a Schematic of the system. The system consists of a broadband light source, a scanning Michelson interferometer with the rapid-scan phase-controlled delay line, a sample gas cell, a photodetector, and a digitizer. The delay line consists of a dispersive element, a focusing element, and a scanning mirror aligned in a reflective $4 \mathrm{f}$-configulation. The broadband light is focused at the Fourier plane in the $4 \mathrm{f}$ system after spectral separation by the dispersive element such that each spectral component is mapped on a different position at the Fourier plane. The scanning mirror changes its angle at an angular frequency of $\omega$ and reflects the light with angled directions. The beam traveling through the $4 \mathrm{f}$ system is retro-reflected with an end mirror and goes back along the same path. The corresponding optical frequency of the pivot position of the scanning mirror in the Fourier plane is indicated as $\nu_{0}$. $\mathbf{b}$ Concept of PC-FTS. The upper part shows how the interferogram is obtained in the time domain. For simplicity, the light fields are depicted as a pulse train with zero carrier-envelope-phase as shown in the pulses in the reference arm. Here, $f_{\mathrm{s}}$ denotes the sampling rate, which may be determined by the pulse repetition rate or sampling rate of the digitizer. At each time frame, the light field in the scan arm acquires a linear spectral phase that is proportional to the angle of the scanning mirror in the delay line. The linear spectral phase ramp adds a group delay and phase delay to the light field. The pivot frequency $\nu_{0}$ is converted to the zero frequency in the down-converted RF spectrum after Fourier-transformation. Therefore, the down-converted spectrum can be positioned inside of the Nyquist range $\left(0-f_{\mathrm{s}} / 2\right)$ by adjusting $\nu_{0}$

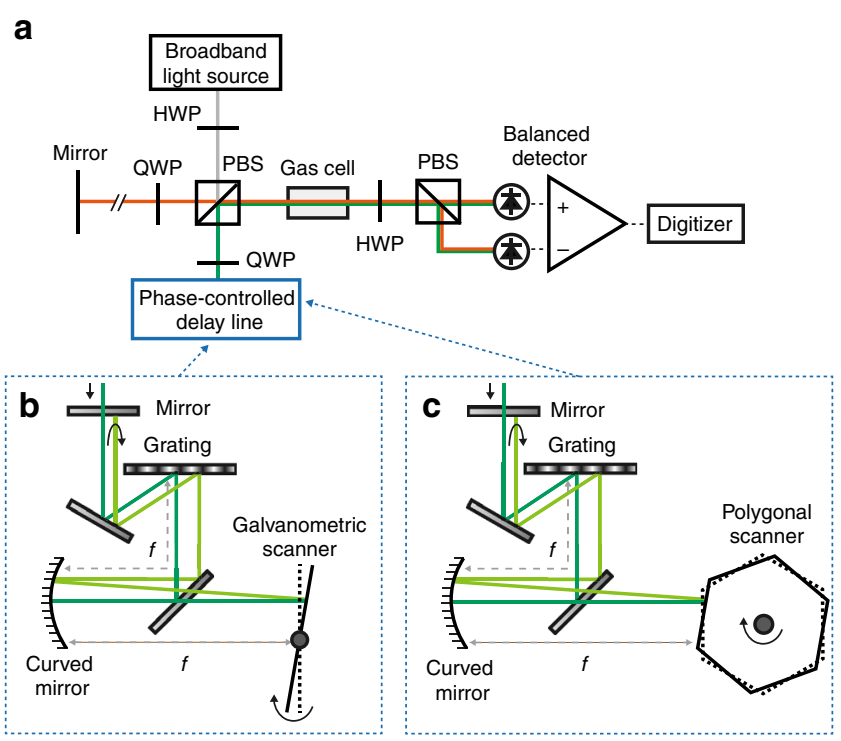

Fig. 2 Detailed schematic of the phase-controlled FTS. a Detailed schematic of PC-FTS. HWP Half-wave plate, QWP Quarter-wave plate, PBS Polarizing beamsplitter. b Phase-controlled delay line with a galvanometric scanner. c Phase-controlled delay line with a polygonal scanner

described in Supplementary Note 1 . The group delay is linearly proportional to time by a factor of $c_{\mathrm{g}}$, while the group velocity can be described as $c_{\mathrm{g}} c$, where $c$ denotes the speed of light. This indicates that a high angular frequency of the scanning mirror makes possible a large bandwidth of the down-converted RF spectrum, leading to an efficient use of the Nyquist range.
Adjusting the angular frequency of the scanning mirror, which determines the down-conversion factor of FTS, is analogous to adjusting the difference in repetition rate between the combs in DCS. Equation 1 tells us that a down-converted RF spectrum has a frequency of $c_{\mathrm{g}}\left(v-v_{0}\right)$, that can be shifted by changing the pivot position of the scanning mirror $v_{0}$. Adjusting $v_{0}$ allows us to fully utilize the Nyquist range while avoiding the aliasing, which is analogous function as that of the difference in CEO frequency between the combs in DCS. The Nyquist theorem tells us the trade-off relation among the scan rate $f_{\text {scan }}$, spectral bandwidth $\Delta v$, and spectral resolution $\delta v$, which may be described as:

$$
f_{\text {scan }} \Delta \nu \frac{1}{\delta \nu}<\frac{f_{\text {s }}}{2}
$$

where $f_{\mathrm{s}}$ denotes the sampling rate. The detailed description of Inequality 3 is provided in Supplementary Note 2.

Phase and group delay correction. Since the down-conversion factor $c_{\mathrm{g}}$ is not exactly a constant value in time, the phasecontrolled delay lines generate temporally nonlinear phase and group delays, which must be corrected for FTS. The detailed description of the nonlinearity is provided in Supplementary Note 1 . To linearize both the nonlinear phase and group delays, we use continuous-wave (CW) interferograms at two different optical frequencies and follow the correction procedure that is in principle the same as that of $\operatorname{DCS}^{19,38}$. Note that the fixed instrumental nonlinearities of the phase and group delays are corrected in the PC-FTS, while the random temporal fluctuations of the difference in repetition rate and CEO frequency are corrected in the DCS. Since the PC-FTS is an autocorrelation-based technique where random temporal fluctuation is inherently small, 
the correction interferograms are not necessarily measured for every measurement.

We measure the CW interferograms at two different frequencies with external-cavity laser diodes with a linewidth of less than $500 \mathrm{kHz}$ lasing at 195.51 and $194.50 \mathrm{THz}$ for the system with a galvanometric scanner, and 196.41 and $195.51 \mathrm{THz}$ for the system with a polygonal scanner. The lasers' frequencies are measured by an optical spectrum analyzer (AQ6317B, Yokogawa) with the accuracy of $\pm 3 \mathrm{GHz}$. The measured CW interferograms are shown in Fig. 3a. The phase nonlinearity is seen especially in the data measured by the system with the polygonal scanner. The phase delays are extracted from the interferograms and plotted in Fig. $3 \mathrm{~b}$. The group delays are calculated from the phase delays at the two different frequencies as shown in Fig. 3c. The maximum group delay is about $100 \mathrm{ps}$, which corresponds to the spectral resolution of about $10 \mathrm{GHz}$.

Broadband absorption spectroscopy. As a proof-of-concept demonstration of the PC-FTS, we measure broadband absorption spectra of gaseous $\mathrm{HCN}$ by the system with a galvanometric scanner. An Er-fiber mode-locked laser that generates femtosecond pulses at a repetition rate of $50 \mathrm{MHz}$ is used as a light source. A full description of the system is provided in Methods section. The interferograms shown in Fig. 4a display the clear modulations of the molecular free-induction decay. By Fourier-transforming a singlesided interferogram with the aforementioned phase and group delay correction $^{19,38}$ and the conventional phase correction methods ${ }^{39-43}$, a broadband spectrum spanning over $7 \mathrm{THz}$ is obtained at a resolution of $10.1 \mathrm{GHz}$ with a high signal-to-noise ratio (SNR) (Fig. 4b). Sharp absorption lines of the overtone vibrational bands of $\mathrm{HCN}$ are clearly observed. Note that the resolution of $10 \mathrm{GHz}$ corresponds to the maximum optical path length difference of $30 \mathrm{~mm}$ in a conventional FTS, which is achieved only by tilting the scanning mirror in the PC-FTS. The scan rate is $300 \mathrm{~Hz}$, which is not limited by the Nyquist range $(0-25 \mathrm{MHz})$ determined by the pulse repetition rate of the mode-locked laser but the scan rate of the galvanometric scanner.

Nyquist-limited rapid-scan spectroscopy. To fully utilize the Nyquist range and achieve the Nyquist-limited highest scan rate, a polygonal scanner can be used instead of the galvanometric scanner. With the faster rotation speed of the polygonal scanner,
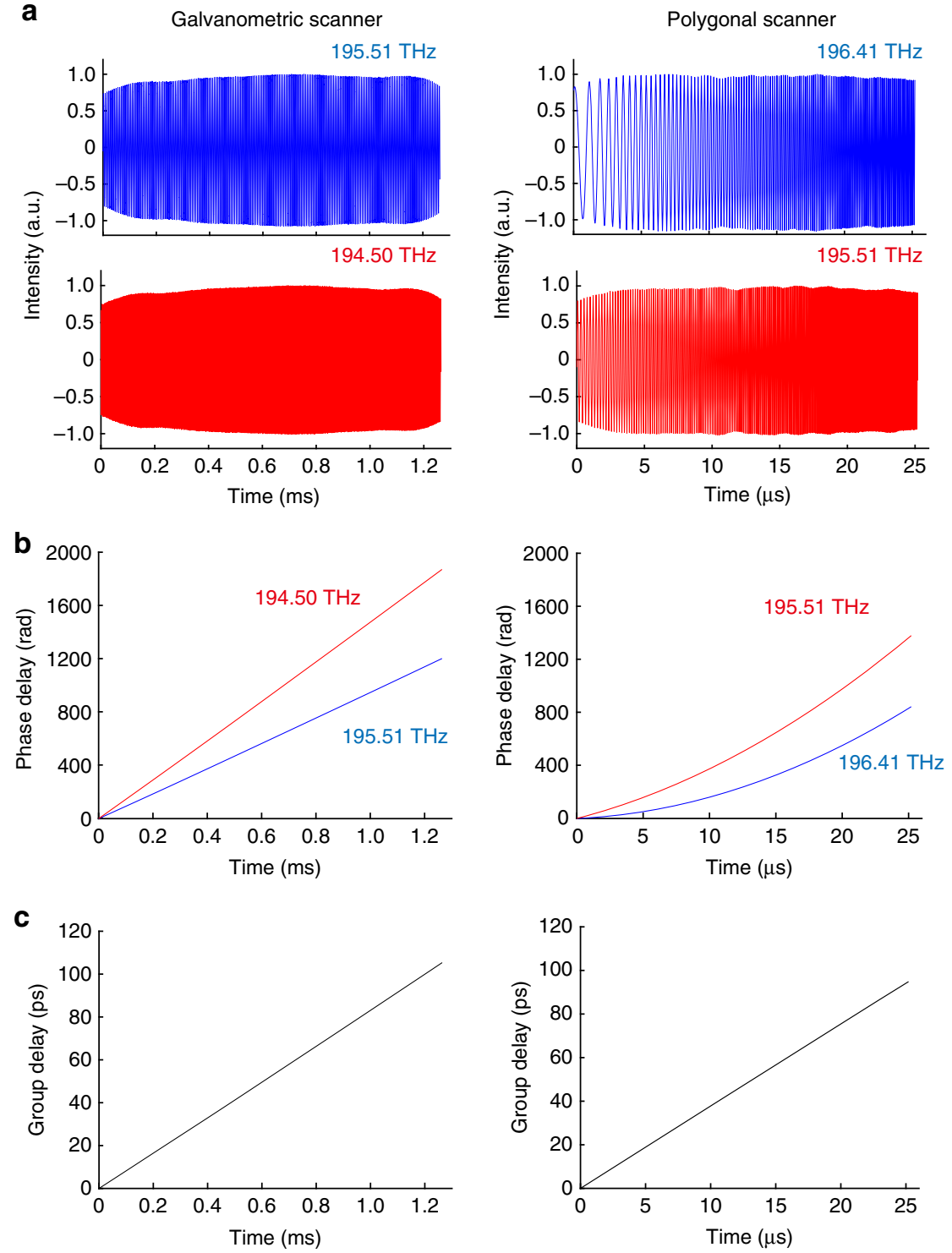

Fig. 3 Characterization of the phase and group delays added by the delay lines. a Measured continuous-wave interferograms at the two different frequencies. b Phase delays of the CW interferograms. c Group delays evaluated from the phase delays at the two different frequencies 

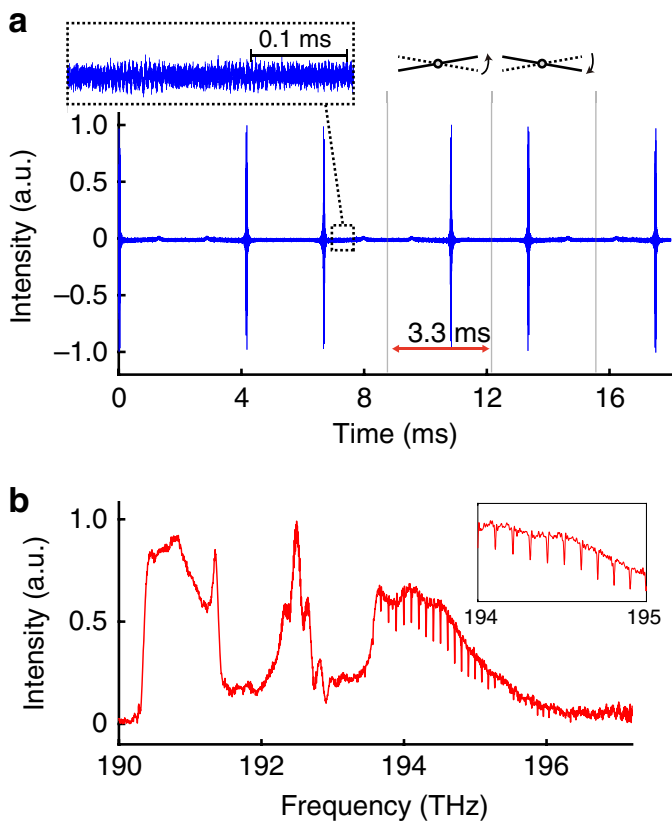

Fig. 4 Broadband spectroscopic measurement by PC-FTS based on a galvanometric scanner. a Continuous interferograms of $\mathrm{H}^{12} \mathrm{C}^{14} \mathrm{~N}$ molecules measured with the mode-locked laser. The single-sided interferograms are continuously obtained at a scan rate of $300 \mathrm{~Hz}$ (the corresponding temporal interval is $3.3 \mathrm{~ms}$ ). The inset shows modulations due to the freeinduction decay. $\mathbf{b} \mathrm{A}$ broadband spectrum obtained by Fourier-transforming a single interferogram. The non-averaged broadband spectrum covering 7 $\mathrm{THz}$ shows absorption lines of $\mathrm{HCN}$ molecules at a spectral resolution of $10.1 \mathrm{GHz}$ with a high signal-to-noise ratio. The structured spectral profile comes from that of the mode-locked fiber laser

a continuous measurement of interferograms at a rate of $12 \mathrm{kHz}$ is demonstrated in Fig. 5a. Figure 5b shows the corresponding consecutive broadband $\mathrm{C}_{2} \mathrm{H}_{2}$ absorption spectra with a bandwidth of $1.5 \mathrm{THz}$ at a spectral resolution of $11.5 \mathrm{GHz}$. The spectra are normalized and converted to transmittance by a baseline fitting with Savitzky-Golay filtering ${ }^{44}$. Note that the effective scan velocity calculated by (scan rate) $\times$ (maximum group delay) $\times c$ is $340 \mathrm{~m} \mathrm{~s}^{-1}$, which is orders of magnitude higher than those of conventional delay lines. The down-conversion factor, (effective scan velocity $) / c=($ scan rate $) \times($ maximum group delay $)$, is about $10^{-6}$. Figure $5 \mathrm{c}$ shows a 20 -averaged spectrum compared to a computationally retrieved spectrum based on HITRAN database $^{45}$. The SNR of a single spectrum is 54 , which is limited by detector noise in this proof-of-concept demonstration. It could be improved by using a detector with better noise performance. The detailed discussion on SNR is provided in Supplementary Note 3.

PC-FTS with an incoherent light source. Since the PC-FTS is an autocorrelation-based technique, it functions as a passive spectrometer with a temporally incoherent light source. Furthermore, since a repetition rate is not usually defined for an incoherent light, the Nyquist frequency of the measurement is determined by an electronic sampling rate of a digitizer. This enables a higher scan rate with a larger Nyquist range.

To show the applicability of an incoherent light to the PC-FTS, we replace the mode-locked laser by a commercially available SLD and perform a proof-of-concept measurement. We acquire HCN absorption spectra over $1.8 \mathrm{THz}$ with a spectral resolution of $11.5 \mathrm{GHz}$ at a scan rate of $24 \mathrm{kHz}$, which is higher than that of the mode-locked laser by a factor of two. Note that the scan rate of $24 \mathrm{kHz}$ is not achievable with the mode-locked laser at the repetition rate of $50 \mathrm{MHz}$ because of the aliasing effect caused by the Nyquist constraint due to the pulse repetition rate. Measured continuous interferograms are shown in Fig. 6a, where freeinduction decay of the molecules is clearly seen every $42 \mu \mathrm{s}$. A spectrum Fourier-transformed from a single interferogram and a 30 -averaged spectrum are shown in Fig. 6b. The fringe observed in each spectrum is the ripple-noise due to the multiplereflections between the facets of the SLD chip.

\section{Discussion}

To see how efficiently the PC-FTS uses the Nyquist range, it is helpful to estimate the bandwidth of the down-converted spectrum when measuring with a conventional FTS. Assuming a commercially available FTS system that can measure a broadband near-infrared spectrum spanning from 176 to $333 \mathrm{THz}$ with a spectral resolution of $7.5 \mathrm{GHz}$ at a scan rate of $1.9 \mathrm{~Hz}$, the downconverted spectrum appears at $45-84 \mathrm{kHz}$, which is much narrower than the Nyquist range set by the sampling rate of a few tens of $\mathrm{MHz}$. The down-conversion factor is $2.5 \times 10^{-10}$, which is orders of magnitude smaller than that of the PC-FTS $\left(10^{-6}\right)$.

Comparing the two Nyquist-limited FTS (DCS and PC-FTS) is useful to see the characteristics of these methods. DCS is a mechanical-scan-free FTS, therefore the system can be robust. Additionally, this frequency comb based FTS can assure high frequency accuracy. On the other hand, PC-FTS works with a single light source, which can be an incoherent light source, making the system simple. Also, this autocorrelation-based FTS can be used as a passive spectrometer.

The concept of this technique can be applied for other wavelengths including the mid-infrared region, where the fundamental vibrational modes of molecules exist. The single-photodetectoroperation of the FTS is especially advantageous in the midinfrared region because of the lack of a high-quality mid-infrared detector-array required for a high-speed dispersive spectrometer. Furthermore, PC-FTS is useful not only for gas-phase molecules but also for liquid-phase or solid-phase materials. As discussed above, since we can arbitrarily choose a combination of the scan rate, spectral bandwidth and resolution, a broader spectral bandwidth, for example, can be measured by reducing the spectral resolution while keeping the scan rate. Finally, this highly efficient FTS technique can also be modified and applied to multidimensional FTS, which requires time-consuming scans of multiple delay lines ${ }^{32}$.

\section{Methods}

Phase-controlled delay line. Our phase-controlled delay line consists of a dispersive element, a focusing element, a scanning mirror, and a flat mirror. These components are aligned to form a reflective $4 \mathrm{f}$ configuration such that a spectrum of the incident light is focused onto the Fourier plane where the scanning mirror is placed. In our demonstration, a reflective ruled grating with 600 lines $\mathrm{mm}^{-1}$ and a curved mirror with a focal length of $150 \mathrm{~mm}$ are used as a dispersive element and a focusing element, respectively. The number of grooves of the grating and the focal length of the curved mirror are to be set for optimizing the system (See Supplementary Note 1 for details). A galvanometric scanner or a polygonal scanner with 36 facets are used as the scanning mirror. The mirror facets of both scanners are coated with gold. The facet size of the galvanometric scanner is $10 \mathrm{~mm}$, and that of the polygonal scanner is $5.6 \mathrm{~mm}$ per each facet. The inner diameter of the polygonal scanner is $63.6 \mathrm{~mm}$ and its rotation speed is set to be 20,000 or $40,000 \mathrm{rpm}$ (rotations per minute) in our experiments. Replacing these optical components to ones with different parameters allows us to choose different combinations of the scan rate, spectral bandwidth and resolution.

Phase-controlled FTS. A full description of our PC-FTS is shown in Fig. 2. A temporally coherent mode-locked Er-doped fiber laser (Femtolite CS-20-GS, IMRA) or an incoherent SLD (S5FC1005S, Thorlabs) is used as a broadband light source. The broadband light is delivered with a single-mode fiber to a fibercollimator placed at the input port of the spectrometer. For a rapid measurement with the polygonal scanner, the light is optically band-pass-filtered with a span of $14 \mathrm{~nm}$ (corresponding to $1.8 \mathrm{THz}$ ) centered at $1533.5 \mathrm{~nm}$ (FBH1550-12, Thorlabs). The collimated light is split into two beams with a half-wave plate and a polarizing 

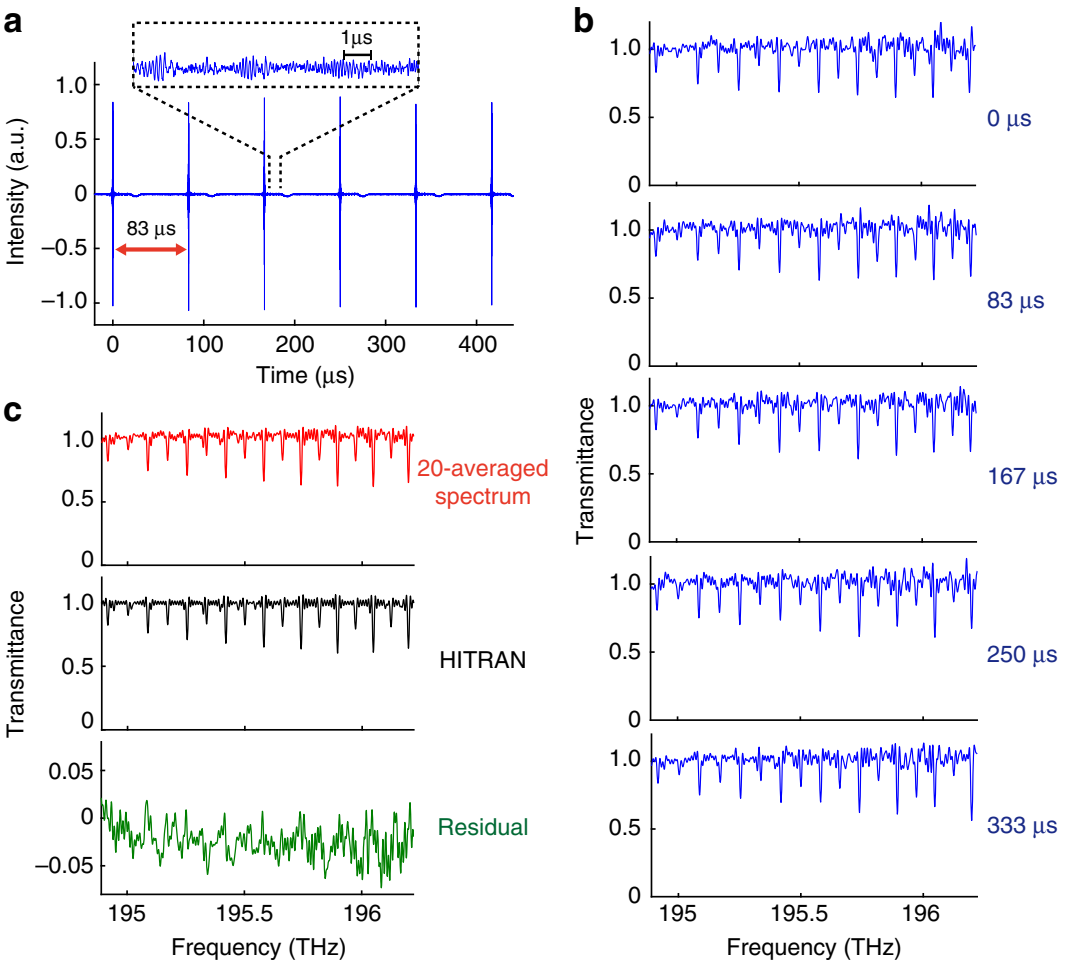

Fig. 5 High-scan-rate broadband spectroscopic measurement by PC-FTS based on a polygonal scanner. a Continuous interferograms of ${ }^{12} \mathrm{C}_{2} \mathrm{H}_{2}$ molecules measured with the mode-locked laser. The burst of the interferogram appears every $83 \mu \mathrm{s}$, corresponding to a scan rate of $12 \mathrm{kHz}$. Clear modulations of the molecular free-induction decay are observed. b Non-averaged transmission spectra corresponding to the interferograms shown in Fig. 5a. Each spectrum covers over $1.5 \mathrm{THz}$ with a resolution of $11.5 \mathrm{GHz}$. The absorption lines of $\mathrm{C}_{2} \mathrm{H}_{2}$ are clearly observed. The noise in the spectra can mainly be attributed to the side-modulations of the sinc function caused by the rectangular Fourier-transform windowing. Apodization (not applied here) can reduce the sidemodulations at the expense of the spectral resolution. c Comparison between a 20 -averaged transmission spectrum and a computationally calculated spectrum based on HITRAN database. The standard deviation of the residual is $1.7 \%$
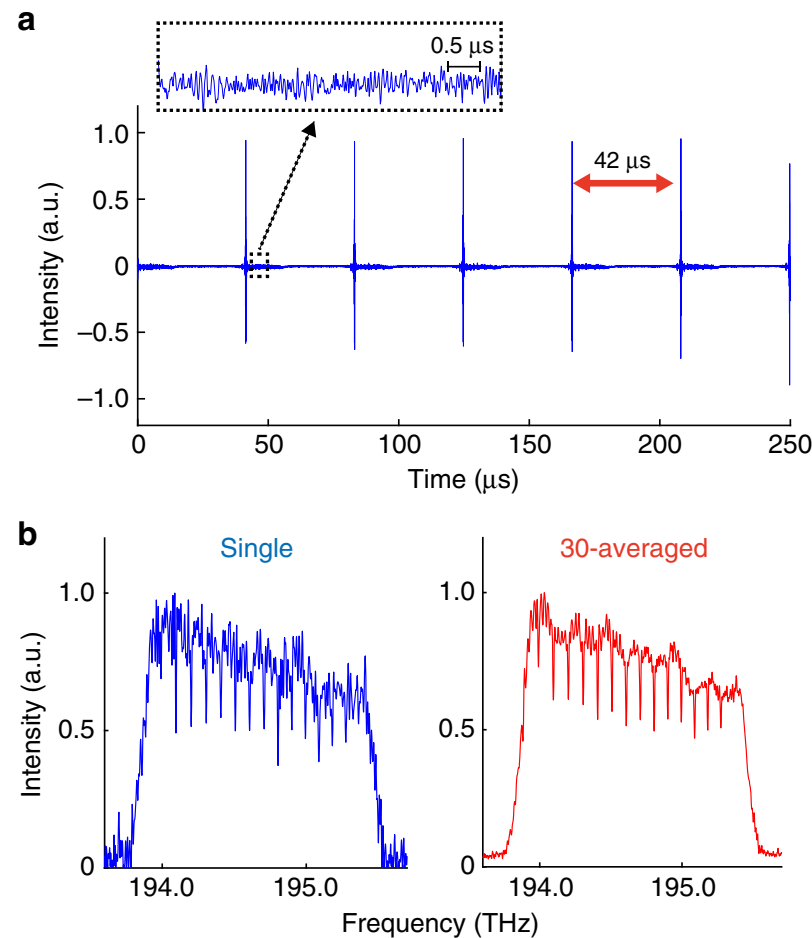

Fig. 6 Interferograms and spectra measured by the PC-FTS with the SLD. a Continuous interferograms of $\mathrm{H}^{12} \mathrm{C}^{14} \mathrm{~N}$ molecules measured with the SLD at a scan rate of $24 \mathrm{kHz}$. $\mathbf{b}$ Spectra obtained by Fourier-transforming a single interferogram (left), and a 30-averaged spectrum beamsplitter. The rotation angle of the half-wave plate is set to have a power ratio of 50:50 between the two output beams of the interferometer. While the beam in the reference arm is retro-reflected by a flat mirror, the other beam in the scan arm travels through the phase-controlled delay line and retro-reflected with a time delay. A quarter-wave plate inserted in each arm rotates the polarization of the reflected beam by 90 degrees, so that the recombined beams exit from the output port of the interferometer with orthogonal polarizations. The beams go through a gas cell containing either $\mathrm{H}^{12} \mathrm{C}^{14} \mathrm{~N}$ (TT-HCN-1OOT-G-Q, triad technology) or ${ }^{12} \mathrm{C}_{2} \mathrm{H}_{2}$ (TT-CH12-50T-G-Q, triad technology) in a two-way (round trip) geometry and detected by an InGaAs balanced photodetector (PDB415C-AC, Thorlabs). For balancing the photodiodes' signals, a half-wave plate and a polarizing beamsplitter are used in front of the detector. The average power of the detected light is set to be $15 \mu \mathrm{W}$ for each photodiode to avoid effects of detector nonlinearity. The detector signal is lowpass-filtered at $21 \mathrm{MHz}$ when using the mode-locked laser. The signal is digitized by a data acquisition board (ATS9440, AlazarTech) at a sampling rate of $125 \mathrm{MS} \mathrm{s}^{-1}$. The digitized time domain data is segmented into independent interferograms and each of them is Fourier-transformed after the phase correction.

Code availability. The Matlab code used for analyzing the data of this study are available from the corresponding author upon reasonable request.

\section{Data availability}

The data that support the findings of this study are available from the corresponding author upon reasonable request.

Received: 25 June 2018 Accepted: 28 September 2018 Published online: 25 October 2018

\section{References}

1. Griffiths, P. R. \& De Haseth, J. A. Fourier Transform Infrared Spectrometry (John Wiley \& Sons Inc., Hoboken, 2007).

2. Cossel, K. C. et al. Gas-phase broadband spectroscopy using active sources: progress, status, and applications. J. Opt. Soc. Am. B 34, 104-129 (2017). 
3. Movasaghi, Z., Rehman, S. \& Rehman, I. U. Fourier transform infrared (FTIR) spectroscopy of biological tissues. Appl. Spectrosc. Rev. 43, 134-179 (2008).

4. Geibel, M. C., Gerbig, C. \& Feist, D. G. A new fully automated FTIR system for total column measurements of greenhouse gases. Atmos. Meas. Tech. 3, 1363-1375 (2010).

5. Gisi, M. et al. XCO2-measurements with a tabletop FTS using solar absorption spectroscopy. Atmos. Meas. Tech. 5, 2969-2980 (2012).

6. Kranendonk, L. A. et al. High speed engine gas thermometry by Fourierdomain mode-locked laser absorption spectroscopy. Opt. Express 15, 15115-15128 (2007)

7. Werblinski, T., Fendt, P., Zigan, L. \& Will, S. High-speed combustion diagnostics in a rapid compression machine by broadband supercontinuum absorption spectroscopy. Appl. Opt. 56, 4443-4453 (2017).

8. Griffiths, P. R., Hirsche, B. L. \& Manning, C. J. Ultra-rapid-scanning Fourier transform infrared spectrometry. Vib. Spectrosc. 19, 165-176 (1999).

9. Süss, B., Ringleb, F. \& Heberle, J. New ultrarapid-scanning interferometer for FT-IR spectroscopy with microsecond time-resolution. Rev. Sci. Instrum. 87, 063113 (2016)

10. Häber, T., Schmitt, U. \& Suhm, M. A. FTIR-spectroscopy of molecular clusters in pulsed supersonic slit-jet expansions. Phys. Chem. Chem. Phys. 1, 5573-5582 (1999)

11. Häber, T., Schmitt, U., Emmeluth, C. \& Suhm, M. A. Ragout-jet FTIR spectroscopy of cluster isomerism and cluster dynamics: from carboxylic acid dimers to N2O nanoparticles. Faraday Discuss. 118, 331-359 (2001).

12. Manning, C. J., Palmer, R. A. \& Chao, J. L. Step-scan Fourier-transform infrared spectrometer. Rev. Sci. Instrum. 62, 1219-1229 (1991).

13. Fleisher, A. J. et al. Mid-infrared time-resolved frequency comb spectroscopy of transient free radicals. J. Phys. Chem. Lett. 5, 2241-2246 (2014).

14. Bjork, B. J. et al. Direct frequency comb measurement of $\mathrm{OD}+\mathrm{CO} \rightarrow \mathrm{DOCO}$ kinetics. Science 354, 444-448 (2016).

15. Coddington, I., Newbury, N. R. \& Swann, W. Dual-comb spectroscopy. Optica 3, 414-426 (2016).

16. Ideguchi, T. Dual-comb spectroscopy. Opt. Photonics News 28, 32-39 (2017).

17. Schliesser, A., Brehm, M., Keilmann, F. \& van der Weide, D. W. Frequencycomb infrared spectrometer for rapid, remote chemical sensing. Opt. Express 13, 9029-9038 (2005).

18. Coddington, I., Swann, W. \& Newbury, N. R. Coherent multiheterodyne spectroscopy using stabilized optical frequency combs. Phys. Rev. Lett. 100, 013902 (2008)

19. Deschênes, J. D., Giaccari, P. \& Genest, J. Optical referencing technique with $\mathrm{CW}$ lasers as intermediate oscillators for continuous full delay range frequency comb interferometry. Opt. Express 18, 23358-23370 (2010).

20. Giorgetta, F. R., Coddington, I., Baumann, E., Swann, W. C. \& Newbury, N. R. Dual frequency comb sampling of a quasi-thermal incoherent light source. In Conference on Lasers and Electro-Optics. paper CTuS3 (Optical Society of America, San Jose, 2010).

21. Bernhardt, B. et al. Cavity-enhanced dual-comb spectroscopy. Nat. Photonics 4, 55-57 (2010).

22. Boudreau, S. \& Genest, J. Referenced passive spectroscopy using dual frequency combs. Opt. Express 20, 7375-7387 (2012).

23. Ideguchi, T. et al. Coherent Raman spectro-imaging with laser frequency combs. Nature 502, 355-358 (2013).

24. Rieker, G. B. et al. Frequency-comb-based remote sensing of greenhouse gases over kilometer air paths. Optica 1, 290-298 (2014).

25. Ideguchi, T., Poisson, A., Guelachvili, G., Picqué, N. \& Hänsch, T. W. Adaptive real-time dual-comb spectroscopy. Nat. Commun. 5, 3375 (2014).

26. Villares, G., Hugi, A., Blaser, S. \& Faist, J. Dual-comb spectroscopy based on quantum-cascade-laser frequency combs. Nat. Commun. 5, 5192 (2014).

27. Millot, G. et al. Frequency-agile dual-comb spectroscopy. Nat. Photonics 10, 27-30 (2016).

28. Suh, M. G., Yang, Q. F., Yang, K. Y., Yi, X. \& Vahala, K. J. Microresonator soliton dual-comb spectroscopy. Science 354, 600-603 (2016).

29. Link, S. M., Maas, D. J. H. C., Waldburger, D. \& Keller, U. Dual-comb spectroscopy of water vapor with a free-running semiconductor disk laser. Science 356, 1164-1168 (2017).

30. Minamikawa, T. et al. Dual-comb spectroscopic ellipsometry. Nat. Commun. 8, 610 (2017).

31. Dutt, A. et al. On-chip dual-comb source for spectroscopy. Sci. Adv. 4, el701858 (2018).

32. Lomsadze, B. \& Cundiff, S. T. Frequency combs enable rapid and highresolution multidimensional coherent spectroscopy. Science 357, 1389-1391 (2017).

33. Ycas, G. et al. High-coherence mid-infrared dual-comb spectroscopy spanning 2.6 to $5.2 \mu \mathrm{m}$. Nat. Photonics 12, 202-208 (2018).

34. Muraviev, A. V., Smolski, V. O., Loparo, Z. E. \& Vodopyanov, K. L. Massively parallel sensing of trace molecules and their isotopologues with broadband subharmonic mid-infrared frequency combs. Nat. Photonics 12, 209-214 (2018).

35. Weiner, A. M. Femtosecond pulse shaping using spatial light modulators. Rev. Sci. Instrum. 71, 1929-1960 (2000).

36. Tearney, G. J., Bouma, B. E. \& Fujimoto, J. G. High-speed phase- and groupdelay scanning with a grating-based phase control delay line. Opt. Lett. 22, 1811-1813 (1997)

37. Oldenburg, A. L., Reynolds, J. J., Marks, D. L. \& Boppart, S. A. Fast-Fourierdomain delay line for in vivo optical coherence tomography with a polygonal scanner. Appl. Opt. 42, 4606-4611 (2003).

38. Giaccari, P., Deschênes, J. D., Saucier, P., Genest, J. \& Tremblay, P. Active Fourier-transform spectroscopy combining the direct RF beating of two fiberbased mode-locked lasers with a novel referencing method. Opt. Express 16, 4347-4365 (2008).

39. Forman, M. L., Steel, W. H. \& Vanasse, G. A. Correction of asymmetric interferograms obtained in Fourier spectroscopy. J. Opt. Soc. Am. 56, 59-63 (1966).

40. Mertz, L. Auxiliary computation for Fourier spectrometry. Infrared Phys. 7, 17-23 (1967).

41. Porter, C. D. \& Tanner, D. B. Correction of phase errors in Fourier spectroscopy. Int. J. Infrared Millim. Waves 4, 273-298 (1983).

42. Herres, W. \& Gronholz, J. Understanding FT-IR data processing. Part 2: Details of the spectrum calculation. Intell. Instrum. Comput., Appl. Lab. 3, 10-19 (1985)

43. Davis, S. P., Abrams, M. C. \& Brault, J. W. Fourier Transform Spectrometry (Academic Press, San Diego, 2001).

44. Savitzky, A. \& Golay, M. J. E. Smoothing and differentiation of data by simplified least squares procedures. Anal. Chem. 36, 1627-1639 (1964).

45. Gordon, I. E. et al. The HITRAN2016 molecular spectroscopic database. J. Quant. Spectrosc. Radiat. Transf. 203, 3-69 (2017).

\section{Acknowledgements}

We thank Makoto Kuwata-Gonokami and Junji Yumoto for letting us use their equipment. We are grateful to Faris Sinjab, Venkata Ramaiah Badarla, and Miu Tamamitsu for their critical reading of the manuscript. This work was financially supported by JSPS KAKENHI (17H04852, 17K19071), JST PRESTO (JPMJPR17G2), Advanced Photon Science Alliance (APSA), Research Foundation for Opto-Science and Technology, and The Murata Science Foundation.

\section{Author contributions}

T.I. conceived the work. K.H. and T.I. designed the system. K.H. conducted the experiment and analyzed the data. T.I. supervised the work. K.H. and T.I. wrote the manuscript.

\section{Additional information}

Supplementary Information accompanies this paper at https://doi.org/10.1038/s41467 018-06956-x.

Competing interests: The authors declare no competing interests.

Reprints and permission information is available online at http://npg.nature.com/ reprintsandpermissions/

Publisher's note: Springer Nature remains neutral with regard to jurisdictional claims in published maps and institutional affiliations.

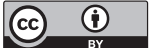

Open Access This article is licensed under a Creative Commons Attribution 4.0 International License, which permits use, sharing, adaptation, distribution and reproduction in any medium or format, as long as you give appropriate credit to the original author(s) and the source, provide a link to the Creative Commons license, and indicate if changes were made. The images or other third party material in this article are included in the article's Creative Commons license, unless indicated otherwise in a credit line to the material. If material is not included in the article's Creative Commons license and your intended use is not permitted by statutory regulation or exceeds the permitted use, you will need to obtain permission directly from the copyright holder. To view a copy of this license, visit http://creativecommons.org/ licenses/by/4.0\%

(C) The Author(s) 2018 